\title{
Corruption et investissement externe direct au Brésil
}

\section{Corrupção e investimento externo direto no Brasil}

Lilian Ransolin de Abreu - Bacharel em Comércio Interancional pela Universidade de Caxias do Sul (UCS). E-mail: liliandeabreu13@yahoo.com.br

Roberto Birch Gonçalves - Doutor em Administração pela Universidade Federal do Rio Grande do Sul (UFRGS). Diretor do Instituto de Pesquisas Econômicas e Sociais (IPES). Email: rbgoncal@ucs.br

Leonardo Roth - Mestre em Administração de Empresas pela Universidade de Caxias do Sul (UCS). Professor do curso de administração e Gestor de protocolo na UCS. E-mail: lroth@ucs.br

\section{Resumo}

O Brasil até 2010 ficou entre os 10 principais países receptores de IED, mas de 2010 a 2014, perdeu posições no ranking dos países receptores de IED. Um dos fatores econômicos que os investidores analisam na escolha do país receptor é a corrupção, que é um dos principais desafios enfrentados pelo Brasil. O presente trabalho tem como objetivo analisar a percepção de executivos de empresas multinacionais sobre o quanto a corrupção influenciou no IED do Brasil no período de 2010 a 2014. Para tanto, utilizouse uma metodologia de pesquisa em duas partes complementares. $\mathrm{Na}$ primeira foram realizadas entrevistas com dois executivos de multinacionais de capital estrangeiro. $\mathrm{Na}$ segunda foram enviados questionários para multinacionais de capital estrangeiro atuantes no Brasil. Após a análise dos dados coletados de ambas as pesquisas percebeu-se que a corrupção é um fator condicionante para a entrada de IED no país e que o Brasil precisa combater e controlar a corrupção, pois a mesma afeta a qualidade dos serviços prestados, a infraestrutura e o investimento global no país.

\section{Palavras-chave}

Investimento Externo Direto. Corrupção. Internacionalização. Brasil.

\section{Résumé}

Le Brésil jusqu'en 2010 était entre les 10 principaux pays récepteurs de IED, mais entre 2010 à 2014, il avait perdu sa position dans le classement des pays récepteurs de IED. L'un des facteurs économiques que les investisseurs avaient analysés c'est à travers le choix du pays récepteur qui est la corruption, qui est aussi l'un des principaux défis qu'on doit faire face au Brésil en ce moment. La présente étude vise à analyser la perception qu'ont les dirigeants d'entreprises multinationales de l'influence de la corruption sur les IED brésiliens de 2010 à 2014. Donc, on avait utilisé une méthodologie de recherche en deux parties complémentaire. Dans la première partie, avaient utilisé des entrevues avec deux exécutifs multinationaux de capital étranger. Dans la deuxième partie avaient été envoyés des questionnaires pour des multinationaux de capital étranger qui sont en fonctions au Brésil. Après avoir fait l'analyse des données capturées des deux recherches, on percevait que la corruption est l'un des facteurs principaux qui avaient causé la rentrée de IED au pays et que le Brésil a besoins de combattre et de contrôler la corruption, alors la corruption elle-même affecte la qualité des services, l'infrastructure et l'investissement global dans le pays.

\section{Keywords}

Investissement externe direct. Corruption. Internationalization. Brésil. 


\section{INTRODUCTION}

Les entreprises en recherche de nouveaux marchés diversifient ses stratégies tant au niveau national qu'international, l'une des options plus importantes d'internationalisation a été un Investissement Externe Direct. Le Brésil selon l'UNCTAD, reste en Gème position dans le classement des pays qui a reçu plus de IED en 2014, reste après de la Chine, Hong Kong, les États Unis, Royaume-Uni et Singapour. La balance du commerce au Brésil en 2015, avait présenté dans les exportations US $\$ 191,1$ milliard et dans les importations $171,5 \$$ milliard, avec un PIB de 1,77\$ Billions (MDIC).

Le pays continue d'être un marché pour les investisseurs externes, alors, dans le classement de destination des Investissements Externes Directs, le Brésil, dans la période de 2010 à 2014, restait entre les 10 pays qui avait reçu plus l'IED au niveau global, et en première position entre les pays de l'Amérique Latine. Mais les flux de IED avait diminué à partir 2011 et la corruption peut être l'un des facteurs qui avaient_promu la réduction du flux d'investissement dans cette période.

La corruption déplace des ressources destinées pour l'infrastructure, la santé et l'éducation, qui sont les points fondamentaux pour le développement du pays. Comme la corruption et une pratique illégale, les cas découverts sont à travers des plaintes et des investigations, ils n'existent pas de preuves qui montrent avec assurance et combien elle représente par année dans le pays ou dans le monde, bien qu'il y ait la perception de son existence, et on sent la présence de sa réflexe qu'il génère dans l'économie du pays.

Dans ce sens, l'objectif de cette recherche est analysé dans sous la perception des dirigeants de multinationales au Brésil quele mesure la corruption au Brésil influençait les niveaux d'Investissements Externes Directs dans la période de 2010 à 2014, en ayant comme objectif de clarifier la question suivante : les niveaux de corruption au Brésil avaient influencé le volume d'investissement externe direct dans la période de 2010 à 2014?

Les résultats de cette recherche montrent que la volatilité de la politique économique adoptée par le Brésil affecte aussi la décision d'investissement externe. En outre, les niveaux de corruption influencent aussi la sécurité et le professionnalisme dans la traitée avec l'investissement externe dans le pays. Les autres facteurs comme l'infrastructure, l'instabilité financière, la bureaucratie ont l'impact fortement sur la décision de l'investissement externe. Cependant, même si ces éléments augmentent les difficultés, ils affectent à peine en partie les investisseurs et non pas la grande partie. 
Il convient de noter que les résultats obtenus sont les résultats des perceptions de deux dirigeants de multinationales (obtenus dans la partie qualitative de l'étude) et de onze répondants (11\%) appartenant aux cent plus grandes multinationales du Brésil (Exame Magazine) obtenu dans la partie quantitative de l'étude.

\section{REVUE DE LITTERATURE}

Le référentiel est structuré en deux parties principales. Dans la première on fera une analyse de l'investissement Externe Direct et sa relation avec les demandes à partir de la globalisation. Dans la deuxième partie on va approfondir sur le thème de la corruption avec transparence comme forme de comprendre quels sont les facteurs qui peuvent être les conséquences majeures dans les analyses, par les entreprises, et dans quelle mesure d'intensité peut-il maintenir ses investissements directs au Brésil.

\subsection{L'INVESTISSEMENT EXTERNE DIRECT}

La globalisation portait de grands changements dans le scénario économique mondial et dans la vie des gens. Selon Cavusgil, Knight et Riesenberger (2010), avec la globalisation, les affaires internationales avaient augmenté de forme surprenante et les flux de capitaux, la connaissance et la technique avaient étendu entre les pays.

Le système financier restait très sophistiqué et la création des organes multilatéraux de réglementation comme l'organisation Mondiale du commerce (OMC) et Fonds Monétaire International (FMI), peuvent être eus un contrôle et une grande coopération entre les nations. Les affaires internationales connectent tous à une économie globale. "les affaires internationales nous donnent l'avantage au accès des biens et services mondiaux et affectent aussi de manière intense notre qualité de vie et bien être économique» (CAVUSGIL; KNIGHT; RIESENBERGER, 2010, P.4.). Para Mariotto (2007), le processus d'internationalisation d'une entreprise va au-delà de l'importation et exportation, car en dépendant de ses objectifs et ressources peuvent être la licence, la franchise, joint venture (l'union des entreprises) et l'investissement direct, l'IED.

Il y a deux types d'investissements: l'investissement portefeuille et l'investissement direct étranger (IED). L'investissement portefeuille c'est l'achat de titres étrangers, où l'investisseur achète avec but d'obtenir un retour financier, sans besoins de contrôler ou gérer et c'est à court terme. Mais l'IDE 
c'est toute contribution d'argent arrivant de l'extérieur qui est appliqué dans la structure productive domestique, dans la forme de participation actionnèrent dans les entreprises existant ou dans la création de nouvelle entreprise (WOLFFENBUT'TE, 2006). Dans les deux entreprises de l'IED c'est le plus qui implique les entreprises, car c'est de long terme et est pratiqué par les grandes entreprises avec assez de ressource et déjà internationalisée (CAVUSGIL; KNIGHT; RIESENBERGER, 2010).

La rentrée de l'IED dans le pays apporte des bénéfices et développements, tel que: i) augmentation de la chaîne productive, régional et international en gérant une augmentation dans les exportations et les importations ; ii) privatisation avec l'introduction de nouvelles technologies et l'augmentation du niveau d'efficacité ; iii) know-how (Savoir-faire) dans les secteurs comme transfert de technologie et formation de capital humain (GREGORY; OLIVEIRA, 2005). Cependant, Moraes (2003) souligne quelques inconvénients comme la faillite de quelques entreprises locales, exode rural, transfert de bénéfice pour les pays des entreprises où se rencontre leur siège social, entre autres.

Selon a UNCTAD (1998), il y a de bonne chance pour qu'un pays attire IED si offre une politique propice pour les activités des transnationales. Les investisseurs vont décider pour le pays qui offre le plus bas prix de transaction, productivité élevée et retour financier. L'ambiant des affaires influence aussi la décision de l'investissement étranger direct. Les taxes corporatives élevées, le retard pour s'ouvrir et fermer une entreprise, le prix élevé des procédures bureaucratiques, le peu de gouvernance corporative, entre autres facteurs, peuvent rendre un pays moins attrayant pour l'investissement externe direct. Les institutions aussi valorisent: un pays démocratique, avec protection légale appropriée à l'infrastructure et peu de corruption tendent à favoriser plus d'entrée d'investissements directs (UNCTAD, 1998).

Les pays développés étaient les plus qui avaient reçu IED dans la décennie de 1990, aucun pays émergeant n’apparaît entre les 10 pays les plus grands. Déjà en 2011 la situation commençait à être modifiée par l'intérêt des investisseurs en pays émergents et en développement. À cause d'une majeure stabilisation politique et économique, le Brésil et la Chine tiennent à se maintenir dans le rang des 10 pays récepteurs de IED dans le monde (MAKSEN, 2013).

Le Brésil est le leader au rang des pays récepteurs de IED dans l'Amérique Latine dans la période de 2010 à 2014, mais en 2014 la région souffrait un déclin au valeur de rentrée de IED. Selon la Cepal (2015) après trois ans consécutifs de grand développement, le ralentissement économique dans la région et les prix des matières premières étaient les responsables qui affectent la rentrée de 
IED dans l'Amérique Latine. Les facteurs qui contribuaient pour cette récession étaient: les conditions macroéconomiques détériorées, le peu d'investissement dans l'infrastructure, la santé et l'éducation fondamentale. La corruption, de 2010 à 2014, est resté comme $6^{\circ}$ indicateur qui perturbe plus les affaires au Brésil (FDC, 2015)

\subsection{CORRUPTION}

La corruption, selon la Transparency International, organisation qui mesure la perception de la corruption mondiale, est définie comme abus de pouvoir confié par les gains privés et ses dimensions (politiques, grandes, moyennes ou petites) et peuvent être classifiées selon combien d'argent perdu et dans quel secteur a eu lieu. Dans le langage commun, le terme «corruption» a un sens très large, en se référant à tout processus de détérioration de valeurs et des pratiques qui accompagnent et analysent telle détérioration. (ABRAMO, 2014).

La corruption n'est pas une chose nouvelle «la corruption politique, comme tout le reste c'est un phénomène historique. Comme telle elle est vieille et mutante» (CARVALHO, 2008, p.237). Longtemps, dans l'Empire brésilien, le terme corruption n'était pas utilisé dans les sujets du gouvernement, parce qu'on croyait du fait que la puissance d'exclusivité du monarque, les actes étaient nuls (SCHWARCZ, 2008).

De Holanda (2016) souligne que les racines de la corruption au Brésil sont culturelles et donc intériorisées dans l'État et au Brésil. Cependant, Souza (2017) élargit la question en affirmant l'existence d'une corruption réelle fortement encouragée par la prétendue «élite économique».

Pour la Transparency International (TI) la corruption punit la société dans sa liberté, la santé et l'argent et se divise en quatre catégories principales: I) dans la politique, l'utilisation de l'argent public en avantage particulier; II) dans l'économie, l'utilisation de l'investissement public en projets de première nécessité comme des écoles, hôpitaux, et routes; III) dans le social, les gens commencent à juger et non confier dans le système politique du pays; IV) l'environnement, qui est sauvagement exploré dû à l'avarice des entreprises. En fait, Bird, MartínezVázquez et Torgler (2008) ont vérifié de manière empirique que la corruption génère moins de pression fiscale, en particulier dans les pays à revenu faible et intermédiaire, contribuant à la cupidité des entreprises.

Kreikebaum (2008) met en évidence que la corruption a deux éléments fondamentaux, qui sont: la subordination et la corruption passive. La subordination assumait une perspective globale en tournant presque impossible 
d'être identifiée par les autorités légales. Autre difficulté c'est d'interpréter les bonus ou paiements supplémentaires dans les entreprises dûes au facteur silencieux dans les transactions. De plus, les employés des entreprises concernées créent leur propre rationalité pour justifier leurs actions. Anand, Ashforth et Joshi (2004) soutiennent que les individus impliqués dans des actes de corruption et la persistance d'actes au fil du temps sont un phénomène qui peut être expliqué en partie par la rationalisation (déni de responsabilité, déni d'intention, déni d’il y a des victimes, équilibre comptable, déni de préjugés sociaux) qui permettent aux employés (et à leurs proches) de voir leurs actions justifiées.

C'est très important de contrôler la corruption, parce qu'elle porte préjudice ou encore une perte à l'efficacité de l'économie. C'est très difficil de prendre son contrôle, car elle est produite secrètement. C'est pourquoi la forme indirecte la plus utilisée sont les indices de perception de corruption de la Transparency International (ABRAMO, 2005).

En concernant à l'organisation non gouvernementale Transparency International calcule annuellement, depuis 1995, l'indice de perception de la corruption (en anglais CPI). Le pays est considéré comme le plus corrompu est celui qui a la note plus près du zéro, quant à le moins corrompu reste plus prêt du cent. Pour arriver dans ces notes, l'ONG a réalisé des recherches annuelles avec des exécutifs et spécialistes des pays évalués, où ces interviewés avait évalué comment ils perçoivent la présence de la corruption dans les institutions publiques dans les pays où ils vivent (FIESP, 2010). Le Brésil, de 2010 à 2014, restait entre $69^{\circ}$ et $73^{\circ}$ dans le classement de corruption comme montre le Tableau 1 ci-dessous.

Tableau 1 - Position de Brésil dans l'Indice de Perception de la Corruption de 2010 à 2014

\begin{tabular}{c|c|c|c}
\hline ANNÉE & CLASSEMENT & POINT & PAYS/NATIONS \\
\hline 2010 & 69 & 37 & 178 \\
\hline 2011 & 73 & 38 & 183 \\
\hline 2012 & 69 & 43 & 176 \\
\hline 2013 & 73 & 42 & 177 \\
\hline 2014 & 69 & 43 & 175 \\
\hline
\end{tabular}

Source: Indices de Perception de la Corruption de la Transparency International (2015)

Dans le classement de 2014, le Brésil était en $69^{\circ}$ dans la liste, restait après le Chili et l'Uruguay (les deux en $21^{\circ}$ position). Le Danemark était leader dans le classement comparativement en 2013 et augmentait sa note pour 43, mais l'idéal serait arrivé à 50, qui est considéré la moyenne, ou encore plus prês de celle-là. 
En 2014, les 175 pays qui étaient évalués, plus de deux tiers restaient avec une note inférieure à 50 (TI,2014).

Dans le Tableau 2, on peut voir les 10 pays que recevaient IED et en quelle position ils se rencontrent dans l'indice de perception de corruption (IT, 2014) et dans l'indice global de compétitivité en 2014 (FDC, 2014). Des 10 pays récepteurs de IED, 7 sont considérés com faible niveau de corruption et avec une bonne collaboration dans l'indice global de compétitivité.

Tableau 2 - Comparation entre IED, IPC et IGC entre les 10 pays en 2014

\begin{tabular}{l|c|c|c}
\hline PAYS & IDE & IPC & IGC \\
\hline $1^{\circ}$ CHINE & 129 & 100 & 28 \\
\hline $2^{\circ}$ HONG KONG & 103 & 17 & 7 \\
\hline $3^{\circ}$ ÉTATS UNIS & 92 & 17 & 3 \\
\hline $4^{\circ}$ ROYAUME-UNI & 72 & 14 & 9 \\
\hline $5^{\circ}$ SINGAPOUR & 68 & 7 & 2 \\
\hline $6^{\circ}$ BRÉSIL & 62 & 69 & 57 \\
\hline $7^{\circ}$ CANADA & 54 & 10 & 15 \\
\hline $8^{\circ}$ AUSTRALIE & 52 & 11 & 22 \\
\hline $9^{\circ}$ INDE & 34 & 85 & 71 \\
\hline $10^{\circ}$ HOLLANDE & 30 & 8 & 8 \\
\hline
\end{tabular}

Source: Auteurs (2015)

Dans un panorama dans les institutions et secteurs affectés par la corruption au Brésil, selon le baromètre de la Transparency International (2013), on trouvait que les politiciens sont vus comme les plus corrompus, suivant les parlementaires et partis politiques. La police et le secteur judiciaire recevaient aussi un grande pourcentage du vote, en les considérant comme des corrompus. Les secteurs de l'administration publique comme santé et éducation aussi étaient considérés aussi dans le rang très élevé de la perception de la corruption.

Une étude faite par la FIESP en 2008 et publiée en mars de 2010, sur le taux de la corruption au Brésil de 1990 à 2008, a conclu que le coût moyen de la corruption est estimé entre 41,5\$ milliard à 69,1\$ milliard (en Reales de 2008) et au PIB estimé entre $1,38 \%$ à $2,3 \%$ entre autres devrait rendre compte que la corruption en période de crise comme le postérieur à 2008 tend à augmenter (RODRIGUEZ, 2016).

La corruption entraîne des coûts de corruption préjudiciable à l'IDE et à la croissance économique. Pour cela, la lutte contre la corruption est une priorité entre les économies émergentes (MORAES, 2003). Ainsi, quand est 
supérieur l'omission dans le contrôle de la corruption, supérieur est la quantité de ressources détournées des activités productives et, donc, supérieure est le prix de la corruption, car ses ressources ne transforment pas en évolution (FIESP, 2010).

Pour combattre la corruption internationale, plusieurs mesures ont été créés par les organisations internationales. Les États-Unis ont créé le Foreign Corrupt Practice Atc qui combat les actes de corruption dans les entreprises américaines; la Chambre du Commerce International a créé les «règles et normes pour combattre «l'extorsion et la subordination»; les Nations unies, la «déclaration contre la corruption et subordination dans les transactions commerciales» et «l'organisation pour la Coopération et la croissance économique (OEDC)»avec plus de 30 économies avancées et quelques pays Latino-Américains que signaient un accord anticorruption (CAVUSGIL; KNIGHT; RIESENBERGER, 2010).

Dans le premier Tableau ci-dessous (TABLEAU 3), sont synthétisés les facteurs, caractéristiques et auteurs en concernant du terme de corruption et investissement externe direct utilisés dans ce référentiel et qu'on avait orienté dans cette recherche qui a été réalisée.

Tableau 3 - Synthèse de la Corruption et Investissement Externe Direct

\begin{tabular}{l|l|l}
\hline \multicolumn{1}{c|}{ FACTEURS } & \multicolumn{1}{|c}{ CARACTERISTIQUES } & \multicolumn{1}{|c}{ AUTEURS } \\
\hline $\begin{array}{l}\text { Investissement } \\
\text { Externe Direct }\end{array}$ & $\begin{array}{l}\text { l'investissement où l'entreprise achète les } \\
\text { produits actifs, comme usine, terrain, capital } \\
\text { entre autres aus extérieurs. c'est à long terme, } \\
\text { en tournant les entreprises transnationales. }\end{array}$ & Marioto (2007) \\
\hline $\begin{array}{l}\text { Attraction/ } \\
\text { Critaires }\end{array}$ & $\begin{array}{l}\text { La structure politique, facilitation pour les } \\
\text { entreprises et déterminantes économiques } \\
\text { comme: recherche du marché, recherche de } \\
\text { ressources actives, recherche d'efficacité. }\end{array}$ & Moraes (2003) \\
\hline Corruption & $\begin{array}{l}\text { Abus de pouvoir confié par des biens privés et } \\
\text { ses dimensions (politiques, grandes, moyennes } \\
\text { ou petites) }\end{array}$ & $\begin{array}{l}\text { Tranparency } \\
\text { International (2015) }\end{array}$ \\
\hline $\begin{array}{l}\text { Les Nations Unies, Banque Mondiale, Fond } \\
\text { monétaires international, police fédérale, } \\
\text { Ministère Public, Recette Fédéral et Cours des } \\
\text { Comptes de l'Union (TCU) }\end{array}$ & $\begin{array}{l}\text { Cavusgil; Knight; } \\
\text { Riesenberger (2010), } \\
\text { Gaetani (2005) }\end{array}$ \\
\hline
\end{tabular}

Source: Auteurs (2015)

En somme pour l'UNCTAD (1998), la corruption n'est pas nécessaire et non plus favorable pour IED, mais pour les pays émergents ou qui sont en train de réformer et améliorer ses politiques de IED cette facilité d'affaire peut rendre grandement difficile le processus. La section suivante décrit la méthodologie utilisée pour enquêter la perception des gestionnaires en concernant de l'IED. 


\section{PROCÉDURES MÉTHODOLOGIQUES}

Le travail est divisé en deux parties l'une qualificative de caractère exploratoire et l'autre descriptive. Dans la première partie on réalisait à travers d'entrevues profondes appuyées par un scénario semi-structuré avec deux exécutifs de multinationales. «On préfère l'utilisation d'entrevues en profondeur, car l'informateur peut fournir des informations assez riches sans perdre l'essentiel» (GIL, 2010, p. 144). L'un des objectifs c'est d'améliorer le questionnaire en captant des informations préliminaires sur le sujet. Le scénario est structuré sur les questions centrales du choix du Brésil par les IEDs matrices, les difficultés, le combat et l'influence de la corruption dans IED.

Dans la première étape les entrevues sont faites par téléphone et sont faite dans les entreprises de Caxias do Sul. Les entreprises sélectionnées sont multinationales de contrôle étranger dans secteur du métal mécanique, la première multinationale est de controle italien et la seconde de controle autrichien. Dans cette étape exploratoire, après avoir transcrit les entrevues, est faite une analyse des contenues en confrontant avec la théorie à travers d'un ensemble de techniques d'analyse de communications (BARDIN, 2004)

Dans la deuxième étape, la méthode utilisée était quantitative, réalisée à travers de questionnaire fermé. Étaient sélectionnées de la revue «Examen Grands et Meilleurs» (Exame Maiores e Melhores), édition de 2014, les 100 plus grands entrepris de contrôle étranger (EXAME, 2014). Une recherche a été faite dans les sites des entreprises et cette recherche a été faite au secteur des relations avec les investisseurs. Après avoir recueilli les courriers ont été envoyés le questionnaire fermé. Deux entreprises demandent l'envoi du questionnaire par courrier. Les données des entreprises et des correspondants étaient maintenues en secret.

Le questionnaire a été envoyé par courrier, et la correspondance par les 100 plus grands multinationaux de capital étranger, para recette, sélectionnés de la revue «Examen Plus grandes et meilleurs» de 2014, avec la finalité d’identifier si la corruption influençait les investissements externes directs dans la période de recherche. Dans le questionnaire, les questions étaient sur l'IED, choix du Brésil, Difficultés, combat à la corruption et l'investissements futur. Les répondants étaient choisis par commodité, c'est-à-dire, ceux qui acceptaient à répondre le questionnaire. Étaient reçues 11 réponses qu'étaient analysées.

Dans l'étape quantitative, est faite une analyse statistique descriptive en considérant les facteurs suivants: IDE, Attraction/Critères, corruption, combat et 
solution. L'analyse statistique descriptive résume les données collectées dans une investigation, synthétisation, classification, vérification s'ils existent de différences entre les groupes en les tournant clair à sa présentation (MALHOTRA, 2001).

\section{PRÉSENTATION DES RÉSULTATS}

\subsection{ANALYSE D'ENTREVUE EXPLORATOIRE}

Dans la première partie d'entrevue étaient sollicités aux interviewés leur fonction, le temps d'entreprise et le secteur (Tableau 4), pour vérifier le niveau de connaissance sur l'entreprise et le sujet abordé.

Tableau 4 - Profil des Interviewés

\begin{tabular}{l|c|c|c}
\hline ENTERVIEWÉ & FONCTION & $\begin{array}{c}\text { TEMPS } \\
\text { D'ENTREPRISE }\end{array}$ & SECTEUR \\
\hline E 1 & Directeur Général & 8 années & Métal mécanique \\
\hline E 2 & Gérent Commercial & 12 années & Métal mécanique \\
\hline
\end{tabular}

Source: Les auteurs (2015)

La première question «L'Investissement Externe Direct est favorable à l'économie d'un pays?» Pourquoi?: les interviewés sont en désaccord, car E1 affirmait “qu'au long des années le profit retourne aux pays d'origine et l'argent sort au Brésil". Quant à E2 affirmait «Oui, c'est favorable, en fonction du prix de capital qui est employé et manque de ressources dans le pays». La vision d'E2 est linéaire avec la partie de théorie qui analyse l'IED sur le point de vue des avantages, principalement aux pays en développement avec le transfert de la technique, modernisation des usines, collection des impôts entre autres (GREGORY; OLIVEIRA, 2005), mais un désavantage c'est la sortie des profits pour la matrice.

Dans la question sur «quels sont les principaux motifs pour le choix du Brésil dans l'IED de la Matrice?» les interviewés étaient d'accord avec les hypothèses d'être présent aux quatre continents avec l'unité de fabrication E1 et le potentiel du marché du Brésil, sa pénurie en équipements, son potentiel naturel, le manque d'infrastructure et son leadership en Amérique Latine (E2). Ce fait soutient Moraes (2003), car le choix du marché dépend des facteurs comme structure politique, facilitation pour les entreprises et déterminants économiques. Le choix du pays peut avoir relation avec la proximité avec les autres marchés qui seront exploités, accès a les ressources naturelles et mains d'oeuvres, entre autres. 
En concernant la question «le choix pour l'installation d'entreprise avait relation avec quelque motivation», les deux ont nié en confirmant que la raison était l'opportunité d'acquisition d'une entreprise à Caxias do Sul, alignée avec les planes stratégies de l'entreprise. Les multinationales choisissent d'acheter le concurrent, car ils ont déjà possédé des clients et fournisseurs, mains d'oeuvre et la localisation stratégique (MERCOSUL).

Quand on questionne si «l'entreprise dans la période de 2010 à 2014, a reçu investissements de la matrice? Et où étaient destinés les investissements ?», les deux interviewés affirmaient qu'ils recevaient investissement dans la modernisation de l'usine et acquisitions des autres entreprises. En effet, la plus grande partie du profit généré par les branches, retourne pour la matrice, mais en même temps elle réinvestit dans les branches, principalement où le pays où elle fonctionne signale croissance ou la possibilité d'acquisition d'un concurrent (MORAES, 2003).

Quand on les questionnait «Quelles mesure le gouvernement brésilien pourrait adopter pour motiver l'attraction de IED dans le pays?», E1 affirmait: «montrer qu’il gouverne de façon sérieuse», quant à E2 répondait: baisser les impôts (taxes), motiver l'investissement, avoir une politique économique claire et à long terme». Les réponses Sont en accord avec l'affirmation de l'UNCTAD (1998), qui dit que les institutions sont importantes, car les investisseurs ont besoin d'avoir la confiance dans le pays dans lequel ils auront investi. Un pays démocratique, avec protection légale appropriée, stabilité économique et politique, rend favorable la rentrée de l'IED.

En concernant «Quels sont les facteurs les plus problématiques pour monter une entreprise au brésil» E1 et E2 possèdent la même logique avec un peu de variation. En somme, Bureaucratie, haute compétitivité, informalité, commerce avec un retour du rendement, basse qualité de mains d'oeuvre et peu d'impôt/taxent (UNCTAD, 1998).

Sur la question «dans son point de vue, les investisseurs voient les niveaux de corruption au Brésil comme un problème pour les entreprises», ils affirmaient l'insécurité (E1), l'instabilité politique et la corruption (E2). La corruption porte incertitudes aux investisseurs et fonctionne comme un impôt sur les entreprises (CASTRO, 2008).

En concernant la question «quelles mesures doivent être prises, à son avis, pour réduire la perception d'impunité des actes des corrompus et combattre la corruption? Quelles institutions qui pourraient faire cette contrôle et lutte». E1 affirmait que le citoyen brésilien devrait élire des gouvernants qui sont vraiment sérieux. Quant à E2 entendait que le gouvernement municipal, d'état et fédéral, 
au-delà du pouvoir législatif, a besoin de combattre la corruption de forme plus énergétique. Alors, le pouvoir judiciaire aura la force pour prendre les positions nécessaires pour combattre les corrompus.

La question clé «à ton avis, dans la période de 2010 à 2014 les niveaux de corruption avaient influencé les IEDs au Brésil? Pourquoi?» les interviewés avaient la perception en affirmant que oui. Non savoir dans quel niveau (E1), mesme les taux de retour aura être bons pour les investisseurs, avec l'aggravation de la situation politique/économique dans les dernières années la tendance c'est la rétractation dans les investissements (E2). En effet, un pays avec une administration publique efficace donne une perception de corruption presque nulle, alors les ressources sont employées et destinées avec sagesse et honnêteté au bien publique (CASTRO, 2003).

\subsection{ANALYSE QUESTIONNAIRE FERMÉ}

L'objectif c'est d'entendre la relation entre l'IDE et la corruption, l'analyse était exécutée en considérant les suivants facteurs: IDE, Corruption, lutte et solution. Le Tableau 5, montre l'identification avec le temps d'entreprise et en quel secteur l'entreprise est en fonction au Brésil.

Tableau 5 - Identification

\begin{tabular}{c|l}
\hline Temps d'Entreprise & \multicolumn{1}{|c}{ Secteur } \\
\hline 2 ans & Chimique et pétrochimique \\
\hline 3 ans & Minéraux \\
\hline 8 ans & Métal mécanique \\
\hline 4 ans & Chimique et pétrochimique \\
\hline 11 ans & Métal mécanique \\
\hline 7 ans & Energie \\
\hline 6 ans & Aciérie et Métallurgie \\
\hline 2 ans & Métal mécanique \\
\hline 7 ans & Métal mécanique \\
\hline 4 ans & Métal mécanique \\
\hline 3 ans & Métal mécanique
\end{tabular}

Source: Les auteurs (2015)

Les répondants sont au secteur de relation avec les investisseurs au groupe des 100 plus grandes entreprises multinationales de capital étranger (EXAME, 2014) et que la plupart des entreprises (54,5\%) sont du secteur de métal mécanique. 


\section{a) IED}

Dans le facteur (Tableau 6) étaient analysé les critères du choix du Brésil para les IED's dans les multinationales; comment les multinationales s'installaient dans le pays; si l'État choisi était dû à quelque proposition; si les multinationales reçoivent propositions pour opérer en autres États; si l'entreprise a fait quelque investissement dans le pays et quels sont les principaux facteurs que rendent difficiles les affaires au Brésil.

Tableau 6 - IDE

Quel est le critère que l'entreprise pour choisir du Brésil par les investissements Externes Direct:

\begin{tabular}{|c|c|c|}
\hline Critère & Répondants & Pourcentage \\
\hline Ressources Naturelles & 1 & $9,1 \%$ \\
\hline Ambient Favorable & 8 & $72,72 \%$ \\
\hline Autres & 2 & $18,18 \%$ \\
\hline \multicolumn{3}{|l|}{ L’entreprise s'installait au Brésil à travers de: } \\
\hline Critère & Répondants & Pourcentage \\
\hline Propre Usine & 5 & $45,45 \%$ \\
\hline Achat Entreprise Local (concurrent) & 4 & $36,36 \%$ \\
\hline Pavillon/Terrain loué & 2 & $18,18 \%$ \\
\hline \multicolumn{3}{|c|}{ L'État choisi par l'installation de l'entreprise avait relation avec: } \\
\hline Critère & Répondants & Pourcentage \\
\hline Motivation fiscale offerte par le gouvernement d'État & 2 & $18,18 \%$ \\
\hline Main-d'oeuvre_spécialisée & 4 & $36,36 \%$ \\
\hline Achat d'entreprise locale & 2 & $18,18 \%$ \\
\hline Localisation stratégique (clients) & 3 & $27,27 \%$ \\
\hline
\end{tabular}

Réfèrent à la compétition entre les États par attraction de IED, l'entreprise dans la période de 2010 à 2014 :

\begin{tabular}{l|c|c}
\hline Motivation & Répondants & Pourcentage \\
\hline $\begin{array}{l}\text { Recevait proposition pour opérer en autre état avec } \\
\text { motivation plus attractive }\end{array}$ & 1 & $9,09 \%$ \\
\hline $\begin{array}{l}\text { L'État où elle opère a offert des incitations à la société pour } \\
\text { rester }\end{array}$ & 2 & $18,18 \%$ \\
\hline Aucune des alternatives & 8 & $72,72 \%$ \\
\hline L'entreprise dans la période de 2010 à 2014 a investi en: & Répondants & Pourcentage \\
\hline $\begin{array}{l}\text { Investissements } \\
\text { Acquisition de concurrent }\end{array}$ & 2 & $18,18 \%$ \\
\hline Ouverture des branches & 6 & $27,27 \%$ \\
\hline Modernisation/Expansion de l'usine & $54,54 \%$ \\
\hline
\end{tabular}




\begin{tabular}{l|c|c}
\hline À son avis, montrent trois facteurs plus problématiques pour faire des affaires au Brésil \\
\hline Facteurs & Répondants & Pourcentage \\
\hline Bureaucratie & 6 & $18,18 \%$ \\
\hline Infrastructure précaire & 5 & $15,15 \%$ \\
\hline Main-d'oeuvre de basse qualité & 1 & $3,03 \%$ \\
\hline Corruption & 1 & $3,03 \%$ \\
\hline Impôt très eleves & 7 & $21,21 \%$ \\
\hline Instabilité économique & 6 & $18,18 \%$ \\
\hline Instabilité politique & 3 & $9,09 \%$ \\
\hline Instabilité monnaie étranger & 4 & $12,12 \%$ \\
\hline Quel conseille serait suggérer à faire l'entreprise au Brésil & \multicolumn{2}{|c}{} \\
\hline Conseille & Répondants & Pourcentage \\
\hline Augmenter les niveaux d'investissement & 2 & $18,18 \%$ \\
\hline Maintenir les niveaux d'investissement & 9 & $81,82 \%$ \\
\hline
\end{tabular}

Source: Auteurs (2015)

Dans le Tableau 6 on peut voir que le critère du choix pour les IED's les majeures parties des multinationales étaient l'ambiant favorable, avec 72,72\%. Les multinationales s'installaient dans le pays à travers de leur propre usine, avec $45,45 \%$ des répondants. Dans l'article l'achat d'entreprise locale et localisation de pavillon ou terrain, le choix de l'état avait relation avec main-d'oeuvre spécialisée, avec 36,36\%, et localization stratégiques (clients, Mercosul) ce qu'on dit en concernant la compétition entre les États. 72,72\% les répondants avaient informé qu'ils n'avaient pas reçu aucun type de motif d'état où opéraient les multinationales. Seulement une recevait motif pour opérer en autre état et deux recevaient motifs fiscaux pour rester dans l'état où ils sont en fonction.

Parmi les investissements faits, 54,54\% des répondants avaient informé que la multinationale avait investi dans la modernisation et expansion de l'usine. Parmi les facteurs plus problématiques trois recevaient la majeur pourcentage: le paiement d'impôt élevé avec 21,21\%, bureaucratie et instabilité économique, les deux avec 18,18\%. et la majorité des répondants conseilleraient à la société mère de maintenir les niveaux d'investissements no Brasil. Il est à noter que les personnes interrogées ne considèrent pas la corruption comme un facteur difficile, ou parce qu'elles ne la perçoivent pas ou ne la perçoivent pas comme un facilitateur. Ainsi, ils ne l'associent pas ni à des problèmes culturels (De Holanda, 2016), ni à des problèmes d'une sorte d'élite (SOUZA, 2017). Cela peut aussi être le résultat de la vision rationalisée de la corruption qui excuse et justifie les corrupteurs pour leurs actes (ANAND; ASHFORTH; JOSHI, 2004). 


\section{b) Corruption}

Dans le facteur de la corruption dans le Tableau 7, était analysé dans quelle mesure la corruption était un problème pour les affaires des investisseurs au Brésil; si les niveaux de perception de la corruption avaient augmenté dans la période de 2010 à 2014 et si la corruption avait influencé les IED's dans cette période.

Tableau 7 - Corruption

À son avis, dans quelle mesure les investisseurs voient les niveaux de corruption au Brésil comme un problème pour ses affaires dans le pays:

\begin{tabular}{l|c|c}
\hline Importance & $\mathrm{N}^{\mathrm{o}}$ de Répondants & Pourcentage \\
\hline Très Important & 3 & $27,27 \%$ \\
\hline Moins Important & 8 & $72,72 \%$ \\
\hline
\end{tabular}

À son avis, dans la période de 2010 à 2014, le niveau de perception de la corruption au Brésil augmentait?

\begin{tabular}{l|c|c}
\hline Augmentait & $\mathrm{N}^{\mathrm{o}}$ de Répondants & Pourcentage \\
\hline Oui & 11 & $100 \%$ \\
\hline A son avis, dans la période
\end{tabular}

À son avis, dans la période de 2010 à 2014, les niveaux de corruption avaient influencé les investissements Externe Direct au Brésil?

\begin{tabular}{l|c|c}
\hline Influence & $\mathbf{N}^{\circ}$ de Répondants & Pourcentage \\
\hline Oui & 10 & $90,91 \%$ \\
\hline Non & 1 & $9,09 \%$ \\
\hline
\end{tabular}

Sources: Les auteurs (2015)

Dans le Tableau 7 on avait vérifié que l'opinion la da majorité des répondants $(72,72 \%)$ que les investisseurs voient les niveaux de corruption comme une médiane pour les affaires au Brésil, mais tous les répondants opinait que les niveaux de corruption avaient influencé les IED's au Brésil de 2010 à 2014. Cela est conforme à l'opinion selon laquelle, au cours de la période post-crisé, en l'occurrence 2008, le niveau de corruption a augmenté (RODRIGUEZ, 2016).

\section{c) Contrôle de corruption}

Dans le facteur de contrõle de la corruption (Tableau 8), était analysé, selon l'opinion des répondants, quelle institution brésilienne qui a plus de confiabilité pour contrôler, combattre et punir les actes des corrompus au Brésil. 
Tableau 8 - Contrôle

\begin{tabular}{|c|c|c|}
\hline \multicolumn{3}{|c|}{$\begin{array}{l}\text { Montre quelle institution brésilienne qui possède plus de confiance dans le contrôle, } \\
\text { combat et punition à la corruption au Brésil actuellement: }\end{array}$} \\
\hline Institution & $\mathrm{N}^{\mathrm{o}}$ de Répondants & Pourcentage \\
\hline Police Fédéral & 8 & $72,72 \%$ \\
\hline Ministère Public & 1 & $9,09 \%$ \\
\hline Tribunal de Comptes de l'Union (TCU) & 1 & $9,09 \%$ \\
\hline Aucune d'elles & 1 & $9,09 \%$ \\
\hline
\end{tabular}

Source: Les auteurs (2015)

Dans le Tableau 8, selon l'opinion de plusieurs des répondants $(72,72 \%)$ la Police fédéral est l'institution plus confiable pour combattre et punir les cas de corruption au Brésil.

\section{d) Action au Combat a la corruption}

Dans l'article «actions au combat à la corruption» ('Tableau 9), était analysé, selon l'opinion des répondants, quelle action la plus efficiente pour combattre la corruption et réduire l'exception d'impunité des actes des corrompus.

Tableau 9 - Actions

\begin{tabular}{|c|c|c|}
\hline \multicolumn{3}{|c|}{$\begin{array}{l}\text { À son avis, quelles sont les actions ci-dessous doivent être prises pour réduire la } \\
\text { perception d'impunité des actes corrompus et combattre la corruption. }\end{array}$} \\
\hline Actions & Répondants & Pourcentage \\
\hline $\begin{array}{l}\text { Plus d'efficace de surveillance et contrôle d'administration } \\
\text { publique. }\end{array}$ & 2 & $18,18 \%$ \\
\hline Justice plus rapide et efficace & 6 & $54,54 \%$ \\
\hline Punition sévère pour les auteurs corrompus & 3 & $27,27 \%$ \\
\hline
\end{tabular}

Source: Les auteurs (2015)

D’après le Tableau 9, l'action qui peut réduire la perception d'impunité et combattre la corruption, pour la majorité des répondants, c'est la justice plus rapide et efficace avec, 54,54\%. Puis de la punition Sévère avec $27,27 \%$, et plus efficace aux surveillance et le contrõle d'administration publique, avec 18,18\%.

\section{CONSIDERATIONS FINALES}

En recherche de nouveaux marchés, de plus en plus, les entreprises sont en train de s'internationaliser, et les Investissements Externes Directs sont plus importants, principalement pour les économies en développement, qui est le cas 
du Brésil, qui en 2014, restait en 7eme position como l'une des plus grandes économies mondiales, et est entre les 10 pays qui ont reçu plus d'investissements. Mais de 2010 à 2014, avec exception de 2012, le pays perdait sa position en plusieurs institutions d'analyse de compétitivité mondiale.

L'objectif de cette recherche était d'analyser à travers du référentiel théorique et des opinions des dirigeants multinationales de capital étranger, si les niveaux de corruption avaient influencé les investissements externes directs dans le pays dans la période de 2010 à 2014.

Les analyses faites dans les deux recherches, tant comme qualitative et quantitative, montrent que les entrepreneurs sont insatisfaits avec la politique économique que le Brésil vient d'adopter dans les derniers jours. Pour la majorité des interviewés, les niveaux de corruption viennent d'influencer la rentrée d'investissement externe dans le pays, car les investisseurs cherchent à destiner ses investissements dans les pays où il y a un meilleur retour financier et une stabilité économique saine à long terme. Cependant, la corruption n'est pas l'une des principales préoccupations des dirigeants, elle vient après le fardeau fiscal, l'infrastructure et la bureaucratie, des aspects essentiellement économiques.

On avait observé aussi que le Brésil a besoins d'améliorer ses indicateurs économiques, à besoins d'investir dans l'infrastructure, réduire l'instabilité économique et financière, tant comme la bureaucratie, en effet on est d'accord avec Rodriguez (2016). Le pays a besoin également réduire et simplifier l'attribution, combattre la corruption et réduire la perception et l'impunité des auteurs et des actes des corrompus. Telles actions sont bénéfiques pour le développement économique du Brésil et pour augmenter l'attraction de IED's, vu que la présence de multinationales de capital étranger contribue na productivité nationale, transfert de la technique, mettre en valor la qualité des produits, en forçant les concurrentes nationales à se moderniser pour pouvoir maintenir les compétitivités. Les multinationales optent aussi pour acheter les concurrentes nationales, en modernisant la structure d'usine et en qualifiant les fonctionnaires, en les beneficiant aussi leur propre intérêt. De plus, s'agissant d'un investissement à long terme, l'IDE est moins enclin à fuir en cas de crise et plus il attire les investissements étrangers, moins l'économie reste fragile.

À cause de cette méthode, l'implémentation d'une politique plus transparente et efficace dans le combat à la corruption serait traitée une grande certitude que les actes corrompus no resteraient impunis, et que les ressources seront bien employées pour la croissance du pays comme un seul corps. 


\subsection{LIMITATIONS D’ÉTUDE ET SUGGESTIONS D’TRAVAUX FUTURS.}

Le manque d’information en référant aux données statistiques des entreprises multinationales de capital étranger qui sont en fonction au Brésil était une des limitations pour le développement de ce travail. Ce n'était pas possible de savoir combien d'entreprises qui sont en fonctions dans le pays et d'où l'État auquel ils sont en fonction. L'autre limitation rencontrée était le peu de disposition des entreprises pour répondre le questionnaire. Beaucoup d'entreprises répondaient en informant qu'elles ne pouvaient pas répondre à la recherche due au thème qui englobe la Corruption. Il convient de noter que les données obtenues reposent sur la perception d'un groupe d'acteurs spécifiques, à savoir des dirigeants de multinationales. La même analyse basée sur la perception d'un autre groupe d'acteurs, tels que les fonctionnaires, conduirait possiblement à des résultats différents.

Pour des recherches futures, on suggère que soient faites des recherches spécifiques de l'économie et/ou régions du Brésil pour vérifier s'il y a de variation en relation au facteur de corruption dans la décision de l'IED. On suggère, aussi, des études périodiques pour vérifier si les actions de combat à la corruption modifiaient l'attraction de IEDs.

\section{BIBLIOGRAPHIE}

ABRAMO, C. W. Corrupção e responsabilidade pública. 2014. Disponible en: <http:/ /www.transparencia.org.br/docs/RespPub.pdf>. Accès le 06 jun.2015.

ANAND, V; ASHFORTH, B. E.; JOSHI, M. Business as usual: The acceptance and perpetuation of corruption in organizations. Academy of Management Perspectives, New York, v. 18, n. 2, p. 39-53, 2004.

AVRITZER, L.; BIGNOTTO, N.; GUIMARÃES, J.; STARLING, H. M. M. Corrupção Ensaios e Críticas, Belo Horizonte: UFMG, 2008.

BANCO CENTRAL. Censo de Capitais Estrangeiros no País - resultados para 2013. Disponible en: <http://www.bcb.gov.br/Rex/CensoCE/port/Censo $\% 20$ 2014\%20ano-base\%202013\%20-\%20resultados.pdf>. Accédé:03 jun.2015.

BARDIN, L. Análise de conteúdo. 3 ed. Lisboa: Edições 70, 2004.

BIRD, R. M.; MARTINEZ-VAZQUEZ, J.; TORGLER, B. Tax effort in developing countries and high income countries: The impact of corruption, voice and accountability. Economic analysis and policy, v. 38, n. 1, p. 55-71, 2008. 
CASTRO, C. Determinantes económicos da corrupção na União Europeia dos 15. Economia Global e Gestão, Lisboa, v.13, n.3, dez. 2008. Disponível em $<$ http://www.scielo.mec.pt/scielo.php?script=sci_arttext\&pid=S0873-7444200 8000300004\&lng=pt\&nrm=iso $>$. Accès le: 09 de jun.2015.

CAVUsGIL, S. T.; KNIGHT, G.; RIESENBERGER, J. R. Negócios Internacionais - Estratégia, Gestão e Novas Realidades, Pearson Universidades, 2010.

CEPAL - Comissão Econômica para América Latina e o Caribe. Ingresso de Investimento Estrangeiro Direto na América Latina e no Caribe. 20082014. 2015. Disponible en: <http://www.cepal.org/sites/default/files/pr/files/ tabla_ingresosied_pt_2015_.pdf>. Accédé:13 jun.2015.

DE HOLANDA, S. B. Raízes do Brasil: edição crítica. Companhia das Letras, 2016.

FIESP - Federação das Indústrias do Estado de São Paulo. Relatório Corrupção: Custos econômicos e propostas de combate. 2010. Disponible en: <http:// www.fiesp.com.br/indices-pesquisas-e-publicacoes/relatorio-corrupcao-custoseconomicos-e-propostas-de-combate/>. Accès le:20 de jun.2015.

FDC. FUNDAÇÃO DOM CABRAL. Global Competitiveness Report 20132014. 2015 Disponible en: <http://www.fdc.org.br/imprensa/Paginas/noticia. aspx?noticia $=40>$. Accédé: 14 jun. 2015.

GATEANI, F. A eficiência do combate à corrupção. Desafios do investimento. Disponible en: <http://www.ipea.gov.br/desafios/index.php?option $=$ com content\&view $=$ article\&id $=771$ : catid $=28 \&$ Itemid $=23>$. Accédé 10 set.2015.

GIL, A. C. Como elaborar projetos de pesquisa. São Paulo: Atlas, 5 ed. 2010. GREGORY, D.; OLIVEIRA, M. F. B. A. O desenvolvimento de ambiente favorável no Brasil para a atração de investimento estrangeiro direto. Rio de Janeiro, 2005. Disponible en: <www.wilsoncenter.org/events/docs/brazil. atracaodeIED.pdf>. Accès le: 06 mai. 2015

KREIKEBAUM, H. Corruption as a moral issue. Social Responsibility Journal, v.4,n.1/2,2008.Disponibleen:<http://dx.doi.org/10.1108/17471110810856857>. Accès le: 06 ago.2015.

KUGLER, M; VERDIER, T.; ZENOU, Y.. Organized crime, corruption and punishment. Journal of Public Economics, v. 89, n. 9-10, p. 1639-1663, 2005. 
MALHOTRA, N. K. Pesquisa de marketing: Uma Orientação Aplicada. 3.ed. Porto Alegre: Bookman, 2001.

MARIOTTO, F. L. Estratégia internacional da empresa. São Paulo: Thomson Learning, 2007.

MAKSEN. Como pode o brasil aumentar a captação de investimento estrangeiro? 2013. Disponible en: <http://docplayer.com.br/1583708-Maksen -como-pode-o-brasil-aumentar-a-captacao-de-investimento-estrangeiro.html $>$. Accès le: 08 jun.2015.

MORAES, O. J. Investimento direto estrangeiro no Brasil. São Paulo: Aduaneiras, 2003.

UNCTAD. United Nations Conference on Trade and Deve. World Investment Report 1998: Trends and Determinats, 1999. Disponible en: <http://unctad. org/en/Docs/wir1998_en.pdf>. Accès le: 09 jun.2015.

POESCHL, G.; RIBEIRO, R. Ancoragens e variações nas representações sociais da corrupção. Análise social, Lisboa, p.419-445, 2010. Disponível em <http:/ /www.jstor.org/stable/41012811?seq=1\#page_scan_tab_contents $>$. Accès le: 06 mai.2015.

POLÍCIA FEDERAL. Operações. Disponible en: <http://www.pf.gov.br/ agencia/estatisticas/operacoes>. Accédé: 01 nov.2015.

PRICEWATERHOUSECOOPERS. Doing business and investing in Brazil. São Paulo: Pricewaterhousecoopers, 2005.

RECEITA FEDERAL. O Investimento ireto Estrangeiro - IDE. Disponible en: <http://www.receita.fazenda.gov.br/aduana/IDE/IDEbrasilCuba/ide.htm>. Accès le: 09 maio 2015.

REVISTA EXAME. As melhores empresas do Brasil em 2014. Disponible en: <http://exame.abril.com.br/negocios/melhores-e-maiores/2014/>. Accès le: 08 ago. 2015 .

RODRÍGUEZ, V. M. C. Una investigación sobre la corrupción pública y sus determinantes. Revista Mexicana de Ciencias Políticas y Sociales, Ciudad de México, Año LXI, núm. 227. pp. 103-136. 2016.

SALA-I-MARTÍN, X.; BILBAO-OSORIO, B.; BATTTISTA, A.; HANOUZ, M. D.; GEIGER, T.; GALVAN, C. The Global Competitiveness Index 2014-2015: Accelerating a Robust Recovery to Create Productive Jobs and Support Inclusive 
Growth. In: SCHWAB, K. (Ed.). The Global Competitiveness Report (20142015). Geneva: World Economic Forum, 2014. Disponible en: < http://www3. weforum.org/docs/WEF_GlobalCompetitivenessReport_2014-15.pdf >. Accès le: 15 set. 2015.

SOUZA, J. A elite do atraso: da escravidão à Lava Jato. Leya, 2017.

SOBEET. Boletim Sobeet. Disponible en: <http://www.sobeet.org.br/index. php?option $=$ com_content $\&$ view $=$ article $\& i d=6 \&$ Itemid $=17>$. Accès le: 06 ago. 2015.

SCHWARCZ, L. M. O Sol do Brasil: Nicolas-Antoine Taunay e as desventuras dos artistas franceses na corte de D. João. São Paulo: Companhia das Letras, 2008.

TRANSPARENCY INTERNATIONAL. Corruption Perception Index. 2014. Disponible en: <https://www.transparency.org/research/cpi/overview>. Accès le: 09 jun. 2015.

Global Corruption Barometer 2013: report. Disponible en: < http:/ / www.transparency.org/gcb2013/report>. Accès le: 06 nov. 2015.

UNCTAD. World Investiment Reports. 1998. Disponible en: < http://unctad. org/en/pages/DIAE/World\%20Investment\%20Report/WIR-Series.aspx $>$. Accès le: 06 jun.2015.

WOLFFENBÜTTE, A. Investimento estrangeiro direto. Desafios do investimento. 2006. Disponible en: <http://desafios.ipea.gov.br/index. php? option $=$ com_content $\&$ view $=$ article $\& i d=2103:$ catid $=28 \&$ Itemid $=23>$. Accès le: 16 set.2015. 
